\title{
TTR
}

Traduction, terminologie, re?daction

\section{La traductologie dans les pays germanophones : état actuel (1996) de la recherche}

\section{Nadja Grbić et Michaela Wolf}

Volume 9, numéro 1, 1er semestre 1996

Le festin de Babel

Babel's Feast

URI : https://id.erudit.org/iderudit/037248ar

DOI : https://doi.org/10.7202/037248ar

Aller au sommaire du numéro

Éditeur(s)

Association canadienne de traductologie

ISSN

0835-8443 (imprimé)

1708-2188 (numérique)

Découvrir la revue

Citer ce compte rendu

Grbić, N. \& Wolf, M. (1996). Compte rendu de [La traductologie dans les pays

germanophones : état actuel (1996) de la recherche]. TTR, 9(1), 279-299.

https://doi.org/10.7202/037248ar d'utilisation que vous pouvez consulter en ligne.

https://apropos.erudit.org/fr/usagers/politique-dutilisation/ 


\section{La traductologie dans les pays germanophones: état actuel (1996) de la recherche ${ }^{1}$}

\section{Nadja Grbić et Michaela Wolf}

Dans les pays germanophones, la traductologie des années 80 se caractérise par une remise en question des modèles théoriques traditionnels. Les positions nouvelles exigent un réexamen général ainsi qu'un élargissement du concept de traduction en introduisant des paramètres tels que " scopos " (Reiß/Vermeer, 1984), " action " (Holz-Mänttäri, 1984) et " culture » (Vermeer, 1990). Ces trois points forts du débat traductologique entraînent par voie de conséquence une critique du concept d'équivalence, celui-ci se voyant ou bien catégoriquement refusé, ou bien fortement relativisé. Dans les publications récentes on subsumerait ce changement de cap par des concepts comme " orientation nouvelle » (Snell-Hornby, 1986), " cultural turn " (Bassnett/Lefevere, 1990) ou « changement de paradigme » (Holz-Mänttäri, 1990).

1. Nadja Grbić et Michaela Wolf de l'Université de Graz (Autriche) inaugurent ici la chronique des ouvrages traductologiques de parution récente en langue allemande. TTR leur sait gré de se faire ainsi les interprètes des chercheurs en théorie et en histoire de la traduction auprès des collègues qui ne connaissent pas suffisamment l'allemand pour avoir accès aux originaux. Nous esperons consacrer à moyen terme des chroniques conçues sur le même modèle aux ouvrages de traductologie en d'autres langues (le russe, l'italien, l'espagnol, le portugais, l'arabe, l'hébreu, etc.). 
What is dominant in the series of new approaches recently presented in Germany [...] is the orientation towards cultural rather than linguistic transfer; secondly, they view translation not as a process of transcoding but as an act of communication; thirdly, they are all oriented towards the function of the target text [...] rather than prescriptions of the source text [...]; fourthly, they view the text as an integral part of the world and not as an isolated specimen of language. These basic similarities are so striking that it is not exaggerated to talk of a new orientation in translation theory. (Snell-Homby, 1990, 82f)

Cette transformation conceptuelle se fait remarquer non seulement par des publications comme Übersetzungswissenschaft - Eine Neuorientierung, mais par d'autres activités telles que le Congrès international de "Translation Studies - An Interdiscipline 》 (Vienne, septembre 1992) ou - à l'occasion de cet événement - la fondation de l' " European Society for Translation Studies (EST) ".

La théorie du " scopos ", l'" agir traductologique " et le transfert de culture ont leur dénominateur commun dans une conception du phénomène de la traduction en tant qu'intégrant les paramètres d'action, de culture et de fonction. Cela n'empêche pas les modes traditionnels d'observation structuraliste-linguistique de maintenir leur statut sans modification. Ainsi se forment deux courants théoriques bien distincts par leurs définitions respectives de l'objet de la traductologie ainsi que de la traduction proprement dite. À l'aide de quelques monographies spécifiques, nous présenterons la façon dont ces discussions se sont poursuivies dans les annés 90 . S'adressant à un public plus large, ces publications donnent un panorama pratique sur les questions les plus importantes. 
Snell-Hornby, Mary et Kadrič, Mira, dir. (1995). Grundfragen der Vbersetzungswissenschaft. Wiener Vorlesungen von Katharina Reiß. Wien, WUV Universitătsverlag (WUV Studienbücher GEWI 1), 132 p., ATS 158.

Par ses études traductologiques, Katharina Rei $\beta$ a effectué un travail de défrichage. Ce sont en particulier ses recherches sur la typologie des textes se référant à la traductologie $(1971,31986)$ selon le modèle de Karl Bühler (1934), suivies par une série de publications et de discussions correspondantes, qui en témoignent, mais c'est aussi l'ouvrage de base rédigé en collaboration avec H.-J. Vermeer, Grundlegung einer allgemeinen Translationstheorie (1984), qui définit la traduction comme une action de transfert culturel. Le texte traduit ne prend sa signification qu'à partir de la définition du but (" scopos ") de l'action de traduction. Les auteurs introduisent le concept d' " adéquation ", qui est défini comme une relation entre le texte-cible et le texte-source, à condition que le " scopos " soit respecté. Ainsi le concept de l'équivalence se voit relativisé comme particularité de l'adéquation.

Les Grundfragen der Übersetzungswissenschafi n'apportent pas de nouveaux résultats, mais ce n'était pas non plus la prétention des auteurs. La plaquette représente la publication d'un cours donné à l'Université de Vienne sous forme de huit conférences portant sur des aspects propédeutiques de la discipline. Par sa préface, son aspect extérieur, son contenu et le prix modeste, la publication vise particulièrement le public estudiantin en premier cycle. À partir de la perspective théorique du scopos, $\mathrm{K}$. Reiß offre un programme de connaissances rudimentaires tout en accompagnant sa présentation de toute une riche gamme d'exemples, c'est-à-dire de textes de différents genres et de nombreux types de traduction. En s'inspirant du modèle de Holmes (1988), l'auteure cherche à baliser le champ de travail de la traductologie, mais elle ne peut pas éviter le fait de s'engager dans une « discussion concernant l'utilité de la théorie ". Cela ne surprendra nullement si l'on considère les débats opiniâtres,

2. Questions générales de traductologie. 
acharnés et sans souplesse qui - dans le pays germanophones - ont eu cours sur la pertinence de la théorie dans les centres universitaires de formation de traducteurs. Une telle situation représente - comme en Autriche, par exemple - la pierre d'achoppement pour de grandes réformes de la formation (voir plus loin le compte rendu de $\mathrm{H}$. Hönig). Au cours de ses conférences dont chacune s'entend comme unité autonome, K. Reiß discute de divers paramètres, tels que l'importance de la compréhension du texte dans le processus de la traduction, le rôle de la pragmatique présenté à titre d'exemple de stratégies de traduction orientées uniquement de façon syntaxiquesémantique, les essais de taxinomie en types et genres de textes ainsi que l'équivalence et l'adéquation comme les concepts-clés de la traductologie. Même si la publication ne constitue pas une introduction exhaustive à la traductologie, elle offre les instruments nécessaires pour effectuer une première abstraction et fournit des possibilités d'une réflexion systématique sur l'activité de traducteur. Bref, un livre qu'on peut mettre - sans réserve - entre les mains de tout débutant.

Ammann, Margret (1995). Kommunikation und Kultur. Dolmetschen und Übersetzen heute. Eine Einfahrung far Studierende. ${ }^{3}$ Frankfurt/M., Verlag für Interkulturelle Kommunikation IKO (thw $=$ translatorisches handeln wissenschaft, vol. 1), 127 p., DM 26,80.

Cela vaut aussi pour le livre Kommunikation und Kultur de Margret Ammann. C'est la troisième édition d'une introduction pour étudiants dont le titre original était Grundlagen der modernen Translationstheorie. Le titre modifié renvoie au point principal que l'auteure fait ressortir dans son introduction: la traduction sera conçue comme acte de communication interculturelle.

Dans le cadre de la traductologie des années 70 et 80 , orientée vers la linguistique, le « Kulturtransfer » est observé surtout

3. Communication et culture. Interprétation et traduction aujourd'hui. Une introduction pour étudiants. 
à partir du " mot "; on s'en tient donc à des éléments isolés pour représenter la spécificité d'une culture. On vise à " combler une lacune lexicale " (Koller, 1992, p. 232) sans établir pourtant un rapport textuel global. Cette approche microstructurelle obstrue la vue sur une perspective globale et macrostructurelle. En revanche, dans le cadre de la théorie du scopos élaborée par K. Reiß et H.J. Vermeer, les deux auteurs défendent la thèse selon laquelle « la traduction signifie un transfert culturel et linguistique. Les cultures et les langues ont respectivement leur propre structure, dans laquelle chaque élément reçoit sa valeur de par sa position par rapport aux autres éléments de la même structure. " (Reiß/Vermeer, 1984, p. 104). Cette thèse est sous-tendue par une conception de la culture comme système sémiotique telle qu'elle est définie, par exemple, dans l'« Anthropologie structurale » de Claude Lévy-Strauss.

Après avoir fourni une brève introduction aux études et à la profession de " traducteur » et en s'appuyant sur la théorie du scopos de H.J. Vermeer, Margret Ammann réussit dans son livre d'une façon claire et insistante - à démontrer les relations qui existent entre communication, traduction et culture. D'abord, elle aborde les éléments de la communication vus sous l'angle de la théorie de l'action, pour $\mathrm{y}$ ajouter les aspects qui se révèlent pertinents pour la communication. À l'aide de nombreux exemples (ainsi que de références littéraires), l'auteure avance la thèse selon laquelle la traduction est agir interculturel dans une situation communicationnelle, et elle s'étend sur les exigences d'un traducteur qui puissent répondre à ces prétentions. En même temps, elle renvoie à l'intégration nécessaire de la théorie et de la pratique dans la traduction tout en soulignant - une fois de plus avec maints exemples à l'appui - la compétence de culture ainsi que la compétence de texte du traducteur. Le contenu du livre, par contre, dépasse fréquemment le sujet de la « Kommunikation und Kultur 》; c'est pourquoi le titre est en quelque sorte trompeur. Il faut rappeler particulièrement la bibliographie, vaste et en partie commentée, qui permet une initiation guidée à la traductologie ainsi qu'à ses domaines connexes. 
Nord, Christiane (1993). Einführung in das funktionale Übersetzen. Am Beispiel von Titeln und Überschriften. Tübingen/Basel, Francke (UTB 1734), 315 p., DM 29,80.

Le titre figurant sur la couverture de ce livre induit le lecteur également en erreur. Apparemment, l'auteure ne veut pas présenter ici une introduction à un champ scientifique. Le sous-titre du travail, qui renvoie au contenu de façon plus concrète n'apparaît qu'à l'intérieur du livre. Cela dit, le lecteur se retrouve devant un travail qui offre des bases solides en théorie et qui, par une multitude d'exemples ainsi que par la transparence de la présentation, rend ses observations et ses inférences immédiatement contrôlables.

Le travail empirique de Ch. Nord s'appuie sur le concept de traduction fonctionnelle. Son interprétation d'une théorie de tendance fonctionnelle se voit présentée aux premières pages du livre. Elle s'en tient à J.H. Vermeer, qui, dans sa " théorie du scopos ", place le " but " au premier rang de la traduction. Une marque significative de cette approche est la fonction du texte-cible, qui peut se distinguer de la fonction du texte-source. Dans ce contexte, on utilise les termes " constance de fonction " et de " changement de fonction ". En tant que théorie générale de la traduction, ce modèle permet de comprendre que le scopos de tout original puisse être formulé sans que les situations concrètes soient prises en considération. C'est dans le cas concret de traduction, pourtant, que $\mathrm{Ch}$. Nord voit des limites à ce postulat. Au même niveau de l'adéquation de fonction, elle place le principe de loyauté, présenté en 1988 (v. aussi Nord, 1991). Cette qualité éthique qu'elle ne veut pas voir confondue avec la "fidélité " au texte-source, maintes fois conjurée, oblige le traducteur à agir avec responsabilité envers ses partenaires, donc envers le client et le récepteur du textecible aussi bien qu'envers l'auteur du texte-source, et donc envers les attentes qui se focalisent dans une traduction réalisée à un moment spécifique et qui se constituent par des conventions spécifiquement culturelles.

4. Introduction à la traduction fonctionnelle. 
$\mathrm{Ch}$. Nord vérifie la viabilité du modèle de la traduction fonctionnelle sur un corpus de plus de 12000 titres puisés dans les domaines des belles lettres, des livres de documentation, des livres d'enfant, des contes, de la poésie et des articles spécialisés. Elle y définit l'unité de traduction " titre " comme un genre textuel propre en l'empruntant à la linguistique du texte après l'avoir vérifié selon des critères de textualité. Dans la deuxième partie du livre, elle analyse, selon les aspects quantitatif et qualitatif, la structure, les fonctions et l'intertextualité des titres dans les espaces linguistiques allemand, anglais, français et espagnol et trouve dans les conventions de ces espaces linguistiques et culturels des priorités chaque fois différentes, qui sont à respecter dans une traduction. Dans le troisième volet, elle présente une systématique des conditions permettant la traduction de titres, une systématique orientée selon leur fonctionnalité, et traite les problèmes de la traduction de titres pragmatiques, spécifiques aux pairs culturels et spécifiques aux pairs linguistiques. Dans la dernière partie, les aspects méthodiques sont repris sous forme de dix-sept thèses. L'étude est non seulement bien documentée et présentée de manière didactique, mais, par la présentation exemplaire de toute la gamme de traduction fonctionnellement orientée et par la circonscription de l'unité textuelle choisie, elle permet aussi une généralisation et une application à d'autres genres de textes.

Gerzymisch-Arbogast, Heidrun (1994). Übersetzungswissenschaftliches Propddeutikums. Tübingen-Basel, Francke (UTB 1782), 190 p., DM 26,80.

Comme le livre de Christiane Nord, la " propédeutique traductologique " éveille chez le lecteur des attentes, qui ne seront pas tout à fait comblées. Le choix du titre semble faire partie de la stratégie du programme de l'éditeur UTB (collection poche université) offrant en particulier des introductions à différentes disciplines. Le titre ne correspond à l'ouvre que dans la mesure où des « Points de vue et philosophies traditionnels » (chapitre 1) sont

5. Propédeutique traductologique. 
présentés sous un angle critique. Par la suite, en revanche, il ne s'agit pas de fournir, de façon " propédeutique", une introduction au travail scientifique. Le lecteur se voit plutôt confronté à une analyse de perspectives multistructurelles de la traduction. Le processus de la traduction est discuté sur deux plans. Le contexte macrostructurel comprend le texte dans sa totalité; l'analyse de différents critères en fait partie, à savoir le type de texte, la compréhension du texte ou les différences culturelles. Sur le plan microstructurel, sont abordés des aspects particuliers, comme certains problèmes lexico-sémantiques ou même syntaxiques, entre autres la reprise d'une métaphore à telle ou telle place du texte ou la dissolution d'une structure syntaxique compliquée.

Gerzymisch-Arbogast se tient à la traduction du "Lemprière's Dictionary 》 (Lawrence Norfolk), sortie en 1992 en version allemande et qui lui sert de base pour ses réflexions. La traduction, qui a été qualifiée de "cabinet d'épouvante " (Hönig, 1995 , p. 121), causa une forte polémique. Après que plus de 100000 exemplaires ont été vendus en peu de temps, onze traducteurs professionnels ont violemment critiqué la traduction en adressant une lettre ouverte au traducteur, à l'éditeur ainsi qu'au lecteur. Une véritable " guerre entre traducteurs » éclata à laquelle la presse participa vivement. Cette polémique reposa la sempiternelle question de la méthode de traduction: Faut-il traduire de façon " aliénante " ou bien " assimilante " ? Faut-il mettre en valeur le " texte-source » ou le " texte-cible » ? Faut-il traduire de manière " rétrospective» ou " prospective » ? À cette problématique simple, Gerzymisch-Arbogast oppose une conception diffërenciée en élaborant, d'une manière systématique, les conditions indispensables d'une critique objective des traductions.

Dans la complexité de sa présentation, l'œuvre s'avère trop exigeante pour les étudiants. L'analyse systématique visant les décisions du traducteur au cours de la traduction d'un côté et, de l'autre, celles du critique de la traduction représente en tout cas une continuation logique des résultats de recherches actuels (p. ex. de la typologie de textes élaborée par $K$. Reiß, qui est intégrée de façon détaillée). Pourtant, l'absence d'un index des noms propres et des 
matières ne facilite pas la lecture, déjà assez compliquée dès le départ.

À côté des cuvres propédeutiques dont il a déjà été question ici et qui peuvent être subsumées sous le caractère " fonctionnel " selon leur approche, nous évoquerons encore une autre introduction (également un succès commercial) qui trouve son origine dans le deuxième courant mentionné plus haut. Il s'agit de la quatrième édition entièrement revue par Werner Koller de Einführung in die Ubersetzungswissenschaft (1992), pendant une longue période l'œuvre standard des centres de formation. W. Koller interprète la traductologie comme discipline subordonnée à une linguistique contrastive et descriptive (en privilégiant pourtant - par rapport à la première édition - les aspects pragmatiques, rhétorico-stylistiques et socio-culturels) et continue à s'appuyer sur la discussion du concept allemand controversé d'équivalence (voir Snell-Hornby, 1988). À l'exemple de W. Koller, R. Stolze (1992) reste fidèle à ses origines. Dans sa discussion de l'herméneutique, elle prend une position critique envers les approches fonctionnelles.

Stolze, Radegundis (1992). Hermeneutisches Übersetzen. Linguistisches Kategorien des Verstehens und Formulierens beim Übersetzen ${ }^{6}$. Tübingen, Narr (Tübinger Beiträge zur Linguistik 368), 388 p., DM 86.

L'herméneutique en tant que théorie de l'interprétation et de la réflexion sur les conditions et les possibilités de la compréhension ainsi que celles de son articulation linguistique est - par définition - immanente au processus de la traduction. L'intégration de nouvelles tendances, p. ex. les recherches sur la réception, accroît l'importance de l'approche herméneutique pour la traduction.

Cela signifie pour la pratique de la traduction que le textesource doit être compris comme totalité multiperspective avant qu'il (1)

6. La traduction herméneutique. Catégories lingtistiques de la compréhension et de la formulation en traduisant. 
ne soit transposé, suivant les catégories de traduction, à la langue/culture-cible. Fritz Paepcke fut l'un des premiers à essayer de rendre opérante la pensée herméneutique au profit de la science moderne de la traduction (Paepcke, 1986). Il a reconnu qu'une analyse des parties de texte ne mène pas à la compréhension de l'ensemble du texte. En suivant le gestaltisme, il considère le texte à traduire comme une totalité qui représente plus que la somme de ses parties (concernant cette approche holistique, voir aussi SnellHornby, 1988, pp. 27-35).

Dans son livre Hermeneutisches Übersetzen, R. Stolze poursuit cette approche là où elle peut faire ressortir les décisions particulières du traducteur. « La performance de l'herméneutique en tant qu'art de l'interprétation consiste à transmettre une constellation de sens, prise dans un autre monde, au monde propre, de façon qu'elle soit comprise. » (p. 46) Pour R. Stolze, traduire ne signifie pas transférer - suivant certaines règles - des structures du textesource dans des structures du texte-cible, mais c'est un travail empathique avec les textes tout en les formulant de nouveau.

Les instruments qui lui servent à mettre en place son argumentation sont entièrement empruntés à la linguistique. À l'aide de 18 textes exemplaires, elle revèle des structures pertinentes à la traduction en employant des catégories de plusieurs genres de textes: la thématique, la sémantique, l'analyse lexicale, la pragmatique et la stylistique. Selon $\mathrm{R}$. Stolze, les trois premières catégories peuvent être mises avant tout sur le compte de la phase réceptive de la traduction, les deux dernières catégories sur le compte de la phase productive.

R. Stolze souligne dans son livre son intention de ne tenir compte que des catégories linguistico-formelles de l'herméneutique. Reste à déplorer que la littérature psycholinguistique portant sur les processus de la compréhension soit entièrement absente. Les résultats théoriques, établis par la science de la traduction, ne sont pris en considération qu'en partie dans la discussion du processus herméneutique. Ainsi les travaux de Kupsch-Losereit, qui travaille depuis un certain temps sur les processus de compréhension dans la 
traduction, ne sont point intégrés. Enfin, l'auteure se concentre de plus en plus sur la traduction comme processus de compréhension et de communication tout en essayant de rendre opérants pour la science de la traduction les résultats nouveaux de l'herméneutique en relation avec la recherche sur la communication (v. KupschLosereit, 1990).

C'est une interprétation également linguistique de la traduction que l'on trouve dans le livre de $\mathrm{M}$. Schreiber, axé sur la différentiation et la délimitation du concept de traduction.

Schreiber, Michael (1993). Öbersetzung und Bearbeitung: Zur Differenzierung und Abgrenzung des Übersetzungsbegriffs ${ }^{7}$. Tübingen, Narr (Tübinger Beiträge zur Linguistik 389), 353p., DM 96.

La définition très large du concept de texte, établie par les poststructuralistes, a contribué à ce que, dans la théorie récente de la traduction, le concept de traduction se voie employé souvent au sens le plus large.

Dans les pays anglophones, c'est surtout André Lefevere qui a cherché à élargir le concept de traduction: non seulement toute forme d'adaptation du texte y est englobée, mais dans son concept de " rewriting " sont également compris editing, anthologizing, historiography et criticism (voir Lefevere, 1992, p. 9). Nous y ajouterons l'anthropological writing (Clifford/Marcus, 1986) ainsi que les adaptations artistiques, p. ex. les modèles médiévaux (comme le « Morgante » de Luigi Pulci, à la Renaissance).

Dans son livre, M. Schreiber essaie de délimiter le concept de la traduction « d'autres types de transformation de texte qui sont 'arbitraires' \# (p. 3). Tout en plaidant pour qu'on ne renonce pas à l'investigation scientifique des " adaptations », il réalise pourtant

7. Traduction et adaptation. Différenciation et délimitation du concept de traduction. 
une valorisation hiérarchique qui est nettement en faveur de la " traduction ". Après avoir abordé les questions essentielles de la théorie de la traduction et de l'arrangement, M. Schreiber établit avec maints exemples à l'appui - une typologie differenciée de méthodes concernant l'adaptation interlinguale. La tentative de fournir une liste systématique des différents phénomènes de la transformation de texte (comme l'adaptation " augmentative ", " adaptative " ou " diminutive ") doit être accueillie de façon positive. Il est pourtant à regretter que $\mathrm{M}$. Schneider reste attaché à la terminologie allemande et ne s'occupe pas des formes de " rewriting ", etc. (voir ci-dessus). Des études concernant l'adaptation ont été réalisées également dans d'autres pays (voir p. ex. Koiné, 1992), mais elles se limitent à recycler avant tout la littérature germanophone.

\section{Hönig, Hans (1995). Konstruktives Übersetzen ${ }^{8}$. Stauffenburg Verlag (Studien zur Translation 1), 195 p., DM 36,80.}

Parmi les publications ici présentées, la « Traduction constructive » de H. Hơnig tient une place particulière. Bien qu'il soit conçu aussi d'une manière assez générale et orienté vers une fonctionnalité, le livre s'adresse expressément à un public tout à fait spécifique, à savoir celles et ceux « qui produisent, commandent ou utilisent des traductions » (7). Au profit de ce public, son but est de « démasquer des illusions, finir avec les malentendus et causer un changement de conscience 》 (quatrième de couverture). « Car ce n'est que de cette façon que peuvent être créées les dispositions susceptibles de satisfaire l'exigence d'avoir de bons traducteurs et de bonnes traductions. " Il travaille à partir du modèle théorique de la " coopération " instaurée entre le traducteur et le destinataire (voir Holz-Mănttări, 1986).

Un peu d'histoire: « Translation studies has reached a stage where it is time to examine the subject itself. Let the metadiscussion begin. " C'est ainsi que J. S. Holmes termina son exposé

8. La traduction constructive. 
sur la nature de la science de la traduction qu'il avait présenté en 1972 à l'occasion du " Third International Congress of Applied Linguistics " à Copenhague (Holmes, 1988, p. 79). Dès qu'une discipline scientifique est en train de naître, on peut observer deux phénomènes à sa surface. D'une part, d'innombrables publications plus ou moins longues commencent à apparaître sur le marché; d'autre part, le discours sur la légitimation de la discipline s'intensifie. En ce qui concerne la traductologie, nous avons pu largement constater ces phénomènes épistémologiques dans toutes ses formes et les conséquences qui en ont résulté. Vingt ans se sont écoulé depuis l'appel de Holmes adressé à la communauté scientifique, et la question se pose maintenant de savoir ce que nous avons à recenser aujourd'hui, d'une part pour la délimitation de la discipline, d'autre part pour son établissement. Sans doute la métadiscussion a-t-elle été entamée; cependant, les efforts de légitimation de la discipline face aux organismes universitaires, aux autres disciplines et à la société, mais surtout face aux représentants de la pratique, sont loin d'être terminés. Cela ne surprend pas si l'on observe quels discours circulent dans les centres de formation universitaires. D'un côté, on constate un boom dans la création de nouveaux départements là où il n'existait pas (ou si peu) de centres de ce genre (Ljubljana, 1995, Wolgograd, 1996); de l'autre, pour des raisons économiques et une prétendue absence de tradition, on ferme des instituts (comme en Grande-Bretagne). D'une part, on souhaite accroître la valorisation des plans d'études par un plus grand nombre de matières théorico-réflexives; d'autre part, les " partisans de la pratique » s'élèvent d'une façon quasi idéologique contre les approches scientifiques.

Bien que les scientifiques soient irrités - on les comprend par les sermons de légitimation figurant en guise d'introduction dans presque toutes les grandes publications visant un large public, on ne peut nier que le besoin est encore loin d'être satisfait. Dans ce contexte, il est extrêmement agréable de voir sur le marché une publication qui ne s'empêtre pas dans une tentative de faire à la fois de la recherche scientifique et de l'auto-justification, mais qui se propose, de manière claire et pragmatique, de réaliser un travail de sensibilisation. Le livre de $\mathrm{H}$. Hönig est écrit de façon accessible et 
il semble facile à assimiler. L'auteur se sert de nombreuses métaphores, images et analogies, en introduisant un grand nombre d'exemples pratiques sans hésiter pourtant à prendre des positions critiques. Les métaphores sont peut-être parfois trop insistantes et difficiles à supporter pour l'amateur d'un langage clair, mais, sur ce point, vu l'objectif bien défini de l'ouvrage, l'auteur mérite une certaine indulgence. Le livre comporte une bibliographie selective commentée, dont la publication la plus récente remonte cependant à 1988 .

Comme nous venons de le mentionner, la " métadiscussion 》 de J. S. Holmes est déjà amorcée et elle va bon train. Ces derniers temps, dans les pays germanophones, quelques publications relevant de ce canon sont parues; par leur composition, elles peuvent servir aussi de manuels.

Mais le livre à écrire, dont l'éditeur annonça la sortie et que tout le monde attendit avec curiosité et intérêt, c'est l'œuvre de Radegundis Stolze: Übersetzungstheorien - Eine Einfïhrung (1994). C'est un manuel conçu pour être employé dans le cadre universitaire qui offre une vue d'ensemble des " écoles ", " théories " et « approches » et qui cherche à discuter les influences venant de disciplines voisines ou à défaire des nouds terminologiques. $\mathbf{R}$. Stolze présente sa matière en quatre grandes parties: « Regard sur les systèmes linguistiques ", " regard sur les textes ", " regard sur l'agir " et " regard sur le traducteur ". On y trouvera un grand nombre de noms et d'écoles, qui sont directement ou indirectement liés à la traductologie, mais on en cherchera en vain d'autres, par contre, qui devraient figurer dans une telle publication. Curieusement, il y manque aussi des noms et des écoles relevant des pays germanophones, comme la « Section particulière de recherche sur la traduction littéraire » de Göttingen, qui fait preuve d'une grande activité et produit régulièrement des résultats de recherche. L'approche herméneutique-linguistique propre à l'auteure (voir aussi Stolze, 1992) ne manque pas de colorer l'ensemble de l'ouvrage. Il

9. Théories de la traduction - Introduction. 
ne surprendra donc personne que certains concepts cognitifs en soient exclus. $\grave{A}$ part ces aspects relevant du contenu, on luj reprocha particulièrement son emploi nonchalant de la terminologie, la présentation de certaines informations de seconde main, comme le manque de rigueur chronologique (voir Nord, 1995).

L'ouvrage de H.J. Vermeer Skizzen zu einer Geschichte der Translation ${ }^{10}$ constitue un vaste projet historiographique. Les deux premiers volumes sont déjà parus: Anfänge - Von Mesopotamien bis Griechenland. Rom und das frühe Christentum bis Hieronymus, ainsi que Altenglisch, Altsächsisch, Alt- und Frühmittelhochdeutsch; deux autres volumes sont annoncés pour 1996. À partir de l'« agir traductorique ", l'auteur tente de mettre en évidence les conditions de formation et de tradition relatives aux stratégies occidentales de traduction et d'interprétation. Il y applique sa théorie du scopos aux traductions de l'histoire et, à partir de lả, il vise à en repérer les fils rouges (c'est-à-dire fonctionnels). Les questions dont il s'inspire sont les suivantes: Pourquoi a-t-on traduit comme on a traduit, qu'a$t$-on traduit, comment a-t-on traduit et, enfin, pourquoi y a-t-il toujours un gouffre entre la théorie et la pratique, même chez un seul auteur? Les attitudes d'esprit l'intéressent et son histoire est une histoire des idées de la traduction. L'ouvrage est bien documenté et comporte une bibliographie très importante. Il arrive que la lecture en soit parfois quelque peu éprouvante, en raison des nombreuses notes et digressions.

Voici un grand projet auquel participent de nombreux auteurs de renom venant de différents pays : le Sachwörterbuch der Translationswissenschaft ${ }^{\prime \prime}$ de Heidemarie Salevsky. Ce manuel n'est pas encore terminé, mais, en raison de sa valeur intrinsèque, nous le mentionnerons ici. Il s'adresse aux scientifiques ainsi qu'aux étudiants et souhaite contribuer à la compréhension interdisciplinaire des phénomènes traductologiques en intégrant les différentes écoles.

10. Esquisses pour une histoire de la traduction.

11. Manuel de traductologie. 
En 1000 pages environ sont discutés des concepts pertinents sous forme d'articles de dictionnaire. Les articles qui passent de la traduction de textes religieux à l'interprétation en langage de gestes sont minutieusement structurés. Les nombreuses notes ainsi que les données bibliographiques offrent des informations sur d'autres aspects des phénomènes particuliers. Donc un livre souhaité, que nous attendons avec impatience.

En dernier lieu, nous évoquerons quelques collections, qui se consacrent à la traductologie dans les pays germanophones. La collection la plus récente et la plus prometteuse est Studien zur Translation, dirigée par Mary Snell-Hornby au Stauffenburg Verlag de Tübingen (vol. 1 : Hönig, 1995; vol. 2 : Kaindl, 1995). En tant que forum pour les scientifiques qui s'occupent de questions interdisciplinaires et interculturelles, la collection Studien zur Translation se propose de présenter de nouveaux modèles et concepts dans le cadre de monographies, d'outils de travail, de recueils d'articles et de thèses de doctorat. Le dialogue entre la théorie et la pratique est l'un des propos importants de la collection, tel qu'il le souligne en particulier le volume de H.G. Hönig.

\section{Les Gottinger Beiträge zur Internationalen} Ubersetzungsforschung sont publiés depuis près de dix ans. Depuis 1987, ils paraissent chez l'éditeur Erich Schmidt et forment une partie indispensable de la recherche sur la traduction - avant tout littéraire. À l'Université Georg-August de Göttingen existe depuis 1985 le domaine particulier de recherche " Die literarische Übersetzung ", dans le cadre duquel sont réalisés des projets interdisciplinaires. Les « Beiträge » mettent l'accent sur l'histoire culturelle de la traduction littéraire ainsi que sur les questions interculturelles tout en privilégiant en particulier des approches historiques touchant la traduction littéraire. Un grand nombre des volumes publiés réunissent les actes de colloques internationaux, dont la plupart ont été tenus à Göttingen.

La collection de Forum Modernes Theater témoigne de l'activité de ce domaine particulier de recherche, publiée chez Gunter Narr (Tübingen). Le dernier volume de la collection, qui 
présente des recherches sur les rapports qu'entretiennent les traductions avec les traditions et conventions sociales et théâtrales, est paru en 1995. Les articles des différents volumes ne sont pas une accumulation d'approches hétérogènes, mais des contributions bien harmonisées et pensées.

C'est également chez Gunter Narr (Tübingen) que paraît la collection de Transfer $\rightarrow$ Düsseldorfer Materialien zur Literaturübersetzung. À l'Université de Heinrich-Heine (Düsseldorf) fut créé le cours d'études de diplôme "Literaturübersetzen ". Transfer s'est proposé de diffuser les résultats des travaux ainsi que les activités de recherche.

Il faut aussi mentionner la collection de translatorisches handeln - wissenschaft (thw), publiée au Verlag für Interkulturelle Kommunikation IKO. Les deux directeurs de la collection, Margret Ammann et Hans J. Vermeer, ont pour objectif d'accueillir avant tout des publications représentant l'approche fonctionnelle de la traductologie. Jusqu'à maintenant, les points centraux ont été la spécificité culturelle dans la traduction ainsi que les contributions déjà mentionnées de H.J. Vermeer en histoire de la traduction.

Bref, les récentes publications, parues sur le marché du livre germanophone, s'appuient sur le changement de paradigmes des années 80; elles mettent en évidence, dans le cadre d'une « nouvelle orientation ", des considérations fonctionnelles, axées sur les cultures et fondées sur une théorie de l'action. Malgré le postulat de l'interdisciplinarité, toujours mis en valeur avec insistance, on constate l'absence de certains domaines, qui pourraient être intégrés aux approches dejà citées, permettant de rendre compte de façon plus complète de certaines questions. Par exemple, nous relevons toujours l'absence d'une élaboration profonde des approches anthropologiques-culturelles de la part des États-Unis et de l'Angleterre. Les travaux sur l'idéologie et ses implications dans le processus de la traduction sont aussi plutôt rares. Manquent également des recherches touchant la traductologie féministe. Celleci a pourtant joué un rôle non négligeable dans les pays anglophones 
et francophones. Et même le domaine de la philosophie du langage n'a pas été intégré.

En énumérant ces déficits, nous estimons qu'un autre reproche, dans le cadre des livres traités ici, doit être adressé à la traductologie germanophone: de façon générale et avec une exception relative pour les travaux en anglais (assez bien représentés), les recherches traductologiques non germanophones trouvent un écho fort limité dans les travaux en langue allemande. L'éclatement de cette hégémonie " germano-centrée " serait souhaitable ${ }^{12}$.

\section{Bibliographie}

AMMANN, Margret (1995). Kommunikation und Kultur. Frankfurt/M., Verlag für Interkulturelle Kommunikation.

BASSNETT, Susan and André LEFEVERE, eds. (1990). Translation, History and Culture. London/New York, Pinter.

BÜHLER, Karl (1934). Sprachtheorie. Die Darstellungsfunktion der Sprache. Jena, Fischer.

CLIFFORD, James and George E. MARCUS, eds. (1986). Writing Culture. Berkeley/Los Angeles/London, University of Califomia Press.

GERMZYMISCH-ARBOGAST, Heidrun (1994). Obersetzungswissenschafliches Propädeutikum. Tübingen/Basel, Francke.

HÖNIG, Hans G. (1995). Konstruktives Übersetzen. Tübingen, Stauffenburg.

12. Nous remercions vivement Klaus Ertler pour sa collaboration à la traduction de ce texte. 
HOLMES, James Stratton (1988). « The name and nature of translation studies ", in J. S. Holmes. Translated! Amsterdam, Rodopi, 67-80.

HOLZ-MÄNTTÄRI, Justa (1984). Translatorisches Handeln. Helsinki, Suomalainen Tiedeakatemia.

(1986). "Translatorisches Handeln - theoretisch fundierte Berufsprofile " in M. Snell-Hornby (1986), 348-374

(1990). "Das Transfer-Prinzip 》, in R. Arntz und G. Thome, eds. Übersetzungswissenschaft. Ergebnisse und Perspektiven. Tübingen, Narr, 59-70.

KAINDL, Klaus (1995). Die Oper als Textgestalt. Tubingen, Stauffenburg.

KOINÉ. Quademi di ricerca sulla traduzione e l'interpretazione (1992) " Traduzione e riscrittura ", II(1-2), 263-353.

KOLLER, Werner(1992). Einführung in die Übersetzungswissenschaft. Heidelberg/Wiesbaden, Quelle \& Meyer.

KUPSCH-LOSEREIT, Sigrid (1990). " Sprachlich-konzeptuelle Verarbeitung von Kulturdifferenz in der Übersetzung ", Lebende Sprachen XXXV(4), 152-155.

LEFEVERE, André (1992). Translation, Rewriting and the Manipulation of Literary Fame. London/New York, Routledge.

NORD, Christiane (1988). Textanalyse und Obersetzen. Heidelberg, Groos (engl. U.: Text Analysis and Translation. Amsterdam, Rodopi, 1990).

(1991). " Scopos, Loyalty, and Translational Conventions ", Target III(1), 91-109.

(1993). Einfürung in das funktionale Übersetzen. Tubingen/Basel, Francke. 
(1995). " Rezension: Radegundis Stolze. Öbersetzungstheorien. Eine Einführung ", $M D \ddot{U}$ (Tübingen, Narr) XLI(2), 40-42.

PAEPCKE, Fritz (1986). Im Obersetzen leben. Hg. von Berger/K./Speier, H.-M. Tübingen, Narr.

REIB, Katharina ( $\left.{ }^{3} 1986\right)$. Moglichkeiten und Grenzen der Obersetzungskritik. Munchen, Hueber.

REIB, Katharina und Hans J. VERMEER (1984). Grundlegung einer allgemeinen Translationstheorie. Tübingen, Niemeyer.

SALEVSKY, Heidemarie, ed. (in Vorbereitung). Sachwörterbuch der Translationswissenschaft.

SCHREIBER, Michael(1993). Öbersetzungund Bearbeitung. Tübingen, Narr.

SNELL-HORNBY, Mary, ed. (1986). Obersetzungswissenschaft - Eine Neuorientierung. Tubingen/Basel, Francke.

Benjamins.

(1988). Translation Studies. Amsterdam/ Philadelphia,

(1990). « Linguistic Transcoding or Cultural Transfer? A Critique of Translation Theory in Germany ", in S. Bassnett and A. Lefevere, eds. (1990), 79-86.

SNELL-HORNBY, Mary und Mira KADRIC, eds. (1995). Grundfragen der Ubersetzungswissenschaft. Wien, WUV.

STOLZE, Radegundis (1992). Hermeneutisches Übersetzen. Tubingen, Narr.

(1994). Ubersetzungstheorien. Tubingen, Narr

VERMEER, Hans J., ed. (1990). Kulturspezifik des translatorischen Handelns. Heidelberg, Universităt Heidelberg. 
(1992). Skizzen zu einer Geschichte der Translation. Bd.

1 und 2. Frankfurt/M., Verlag für Interkulturelle Kommunikation.

Nadja Grbic et Michaela Wolf: Institut für Ûbersetzer- und Dolmetscherausbildung, Karl-Franzens-Universitalt Graz, Merangasse 70, A-8010 Graz, Autriche

tw 